\title{
Association of asthma with the risk of acute leukemia and non-Hodgkin lymphoma
}

\author{
MIN-HANG ZHOU and QING-MING YANG \\ Department of Hematology and Oncology, The First Affiliated Hospital of The People's \\ Liberation Army General Hospital, Beijing 100048, P.R. China \\ Received July 23, 2014; Accepted April 22, 2015
}

DOI: $10.3892 / \mathrm{mco} .2015 .561$

\begin{abstract}
An increasing incidence of hematological malignancies has been observed in children and adults worldwide over the last few decades. Asthma is a common chronic inflammatory disease. The aim of the present meta-analysis was to evaluate the potential association between a history of asthma and the risk of acute lymphoblastic leukemia (ALL), acute myeloid leukemia (AML) and non-Hodgkin lymphoma (NHL). A literature search was performed through PubMed and the Cochrane Database of Systematic Reviews and the Newcastle-Ottawa Scale was used to evaluate the quality of the selected studies. The $\mathrm{I}^{2}$ index was used to evaluate heterogeneity and the outcome was measured as the odds ratio (OR) by the random-effects model. A total of 16 case-control studies were included. All the studies were of high quality. The OR for ALL was 0.90 [95\% confidence interval (CI): 0.68-1.19; $\mathrm{P}=0.45 ; \mathrm{I}^{2}=79 \%$ ]. The $\mathrm{OR}$ for $\mathrm{AML}$ was 0.85 (95\% CI: $0.67-1.08 ; \mathrm{P}=0.19 ; \mathrm{I}^{2}=8 \%$ ). The OR for NHL was 0.91 (95\% CI: $0.83-1.00 ; \mathrm{P}=0.05 ; \mathrm{I}^{2}=0 \%$ ). Asthma was found to be inversely associated with the risk of NHL. A negative trend of association of asthma with ALL and AML was also observed. However, additional large prospective studies are required to confirm these findings.
\end{abstract}

\section{Introduction}

Acute leukemia and non-Hodgkin lymphoma (NHL) are the most common malignancies in children and adolescents $(1,2)$, with an increasing incidence observed in children and adults worldwide over the last few decades (3-5). Although ionizing radiation, certain chemotherapeutic agents, certain infections, a family history of disease and certain genetic disorders have been reported to be associated with hematological malignan-

Correspondence to: Dr Qing-Ming Yang, Department of Hematology and Oncology, The First Affiliated Hospital of The People's Liberation Army General Hospital, 51 Fucheng Road, Beijing 100048, P.R. China

E-mail: yangqm@medmail.com.cn

Key words: asthma, acute lymphoblastic leukemia, acute myeloid leukemia, non-Hodgkin lymphoma cies, the etiology of acute leukemia and NHL has not been fully elucidated.

Severe autoimmune conditions, such as systemic lupus erythematosus and rheumatoid arthritis, have been found to be associated with acute leukemia and NHL; however, little is known on the role of subtle immune impairments in the development of diseases, such as asthma. Asthma is an aberrant chronic immune response due to a lack of infections during early childhood (6,7). The association of asthma with acute leukemia and NHL may be based on two hypotheses: One is the immune surveillance hypothesis, according to which the possible mechanism underlying the reduced the risk of asthma patients to develop acute leukemia and NHL is that asthma enhances the ability of the immune system to detect and destroy cancer cells (8). By contrast, the antigenic stimulation hypothesis suggests that immune-stimulating conditions may predispose to cancer, due to an increasing number of random mutations in activated dividing immune cells (9-12). Previous studies suggested that asthma was protective against leukemia and lymphoma (13-16), whereas other studies indicated asthma as a risk factor (17-19), or reported no association (20-23).

Given the conflicting results on the association of asthma with the risk of leukemia and NHL, a meta-analysis was conducted to elucidate this association. The objective of the present study was to evaluate the potential association between a history of asthma and the incidence of acute leukemia and NHL.

\section{Materials and methods}

Literature search. Two authors independently performed a literature search through PubMed and the Cochrane Database of Systematic Reviews through to April 20, 2014. The terms used in the search were 'asthma AND (leukemia or lymphoma)'. The title and abstract of every article were reviewed. According to the inclusion criteria, full-text articles were selected. The reference lists of the full-text articles were also searched for additional relevant studies.

Inclusion and exclusion criteria. An article was considered as useful if it reported original data on the association between asthma and the risk of acute lymphoblastic leukemia (ALL), acute myeloid leukemia (AML) or NHL from epidemiological studies. Only studies published in English were included. The 
articles included were required to provide sufficient information to calculate the odds ratio (OR). Any disagreements regarding inclusion or exclusion of a study were resolved by a joint review with other authors. Only the most recent articles were selected when the same study produced several publications.

Data extraction. Two authors independently performed the data extraction. The extracted data included name of first author, publication year, country, time of diagnosis of acute leukemia or NHL, age range of cases, number of samples (cases, controls, asthmatics and non-asthmatics), methods of assessment of asthma (medical records or interview questionnaires) and matching factors. Any disagreements were resolved by a joint assessment of the original study with other authors.

Quality assessment. Two authors independently evaluated the quality of the selected studies using the Newcastle-Ottawa Scale (NOS). The NOS for case-control studies includes three parameters, namely selection, comparability and exposure assessment. Any individual article may get the maximal NOS score of 4 points for selection, 2 for comparability and 3 for exposure. A NOS score of 1-3 was considered as low-quality, a score of 4-6 as intermediate-quality and a score of 7-9 as high-quality. Any disagreements on the NOS score of a study were resolved by a joint review of the original article.

Data synthesis and analysis. The primary result measured was OR with $95 \%$ confidence interval (CI) of developing ALL, AML or NHL in patients with a history of asthma. The Mantel-Haenszel method and the random-effects model were applied to measure the outcomes $(24,25)$. The $\mathrm{I}^{2}$ index was used to evaluate heterogeneity (26). $\mathrm{I}^{2}$ values of 25,50 and $75 \%$ were considered to reflect mild, moderate and severe heterogeneity, respectively. Meta-analyses were performed for ALL, AML and NHL using the Review Manager 5.2 software (Copenhagen: The Nordic Cochrane Centre, The Cochrane Collaboration, 2012). In the forest plots, OR values $>1$ suggested an increased risk, whereas OR values $<1$ suggested a decreased risk of cases among individuals with a history of asthma.

\section{Results}

Search results. The results of the literature search are summarized in Fig. 1. A total of 668 records were identified during our initial search. Following a review of the titles and abstracts, 618 articles did not pertain to the association between asthma and the risk of acute leukemia or NHL and were excluded. The remaining 50 articles were retrieved for full-text review. Following a detailed review, a further 34 articles were excluded (27 were irrelevant to the association between asthma and the incidence of acute leukemia or NHL, 1 was a case report and 6 were reviews). No additional articles were identified through searching the reference lists of the 50 articles. Finally, 16 case-control studies were included in our meta-analysis.

Characteristics of the studies. The main characteristics of the 16 studies included in our analysis are summarized in Table I. The studies were published between 1996 and 2012 . A total of 7 studies originated from Europe, 6 from North

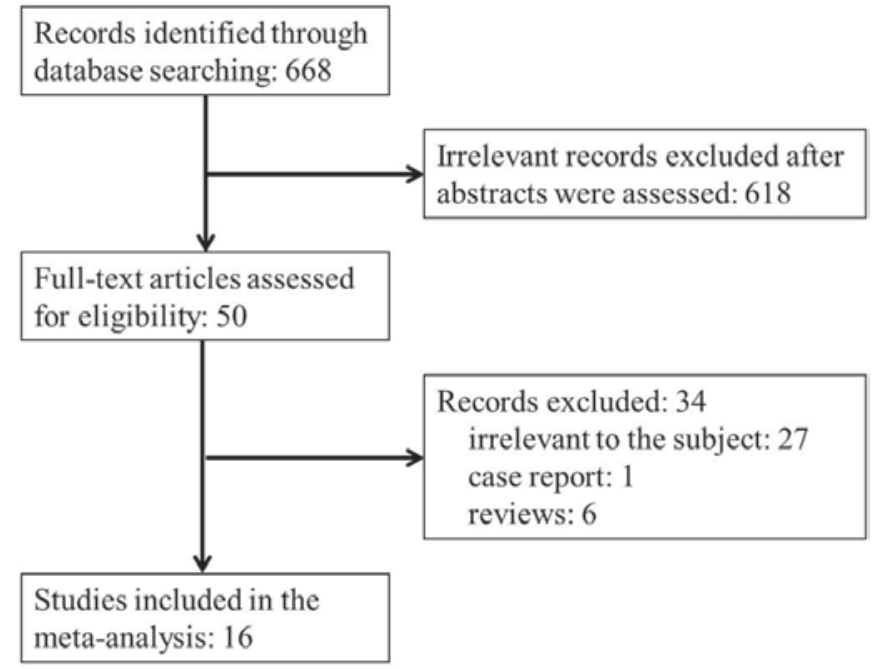

Figure 1. Flow diagram of the study selection process.

America, 2 from Australia and 1 from Asia. The time of diagnosis of the cases was between 1980 and 2008. The cases in the studies were children or adolescents in 9 studies, adults in 6 studies and both in 1 study. A total of 5,738 ALL patients, 4,362 AML patients, 22,048 NHL patients and 168,397 controls were included in our analysis. A history of asthma was identified by interviewing with questionnaires in 12 studies, reviewing medical records in 3 studies and using both methods in 1 study. In all the studies, the controls were matched to the patients by age and gender and 8 studies were adjusted by region of residence. As regards quality assessment, all the included studies were of high quality (NOS score $\geq 7$ ).

Outcome results. A total of 8 studies reported outcomes on the association between asthma and the risk of ALL. The OR for ALL was 0.90 (95\% CI: $0.68-1.19 ; \mathrm{P}=0.45 ; \mathrm{I}^{2}=79 \%$; Fig. 2).

A total of 5 studies provided data on the association between asthma and the risk of AML. The OR for AML was 0.85 (95\% CI: 0.67-1.08; $\mathrm{P}=0.19 ; \mathrm{I}^{2}=8 \%$; Fig. 3).

A total of 8 studies reported data on the association between asthma and the risk of NHL. The OR for NHL was 0.91 (95\% CI: 0.83-1.00; $\mathrm{P}=0.05 ; \mathrm{I}^{2}=0 \%$; Fig. 4).

There was a significantly reduced risk of developing AML in asthmatic patients. In addition, there was an inverse association between asthma and acute leukemia, but this association was not statistically significant.

\section{Discussion}

In our meta-analysis, asthma was found to be associated with statistically significant reductions in the risk of NHL, whereas it was associated with non-statistically significant reductions in the risk of ALL and AML.

A hypothesis has been proposed with regard to the mechanism of the inverse association of asthma with NHL. According to the cancer immune surveillance hypothesis, the immune system identifies and eradicates aberrant cells in the body, thus averting the development of a number of potential 


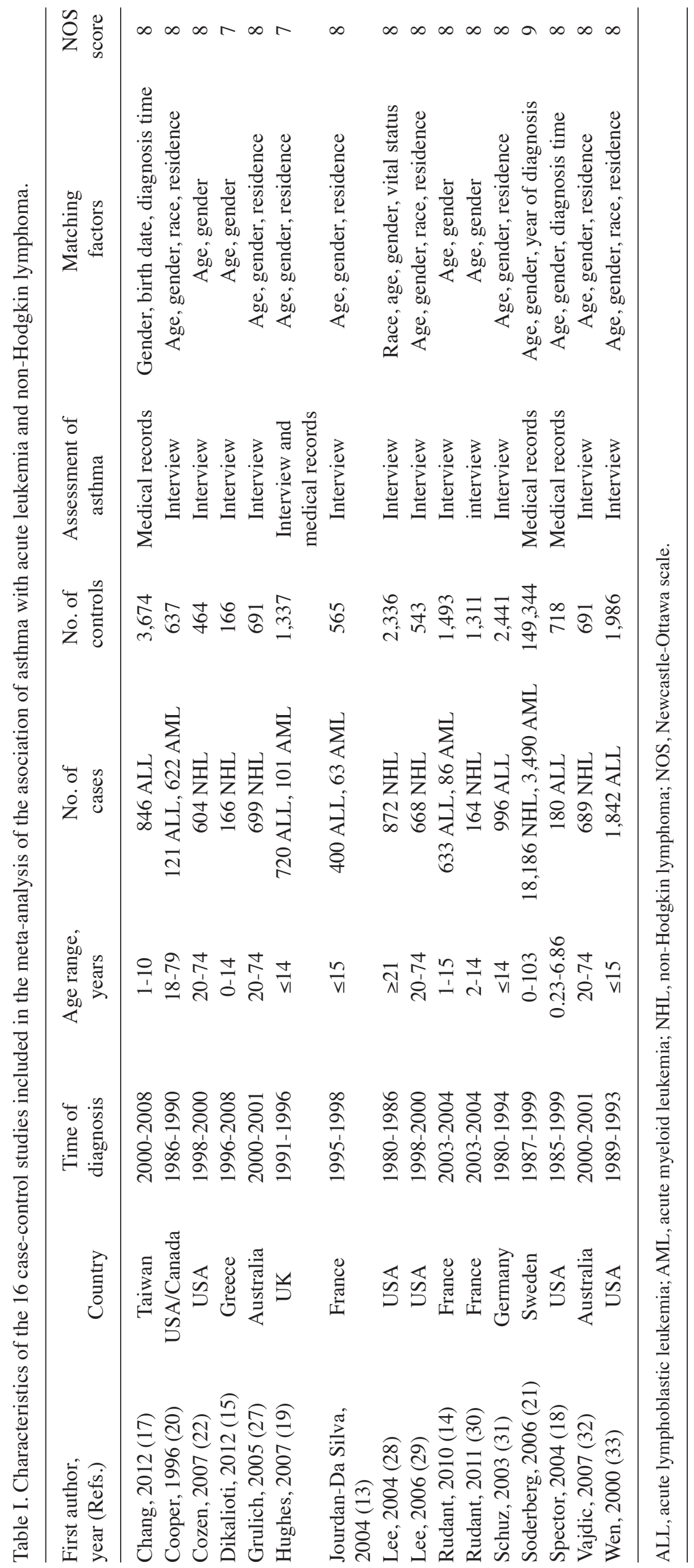




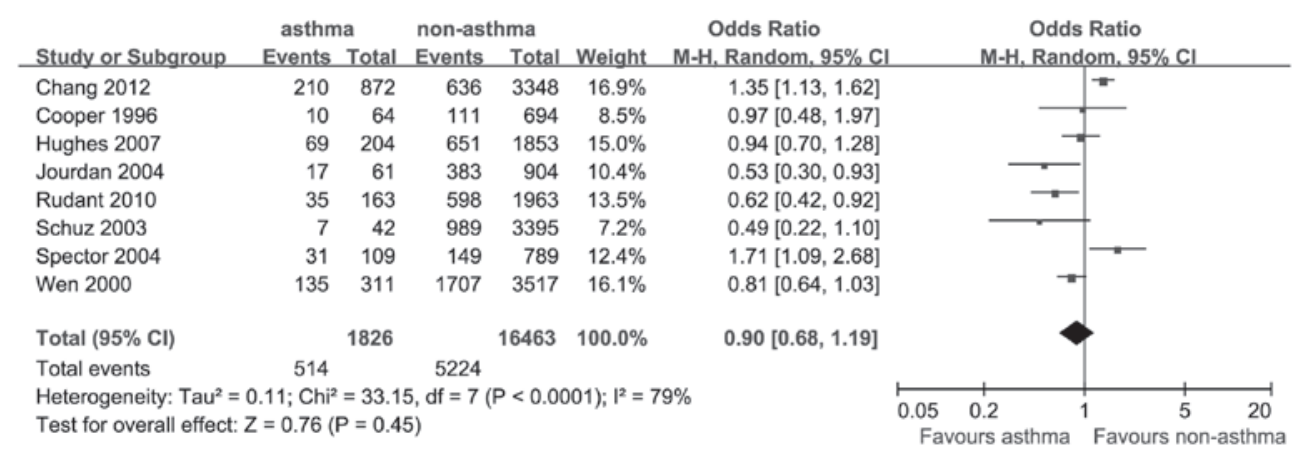

Figure 2. Estimates of the odds ratio of developing acute lymphoblastic leukemia in patients with a history of asthma.

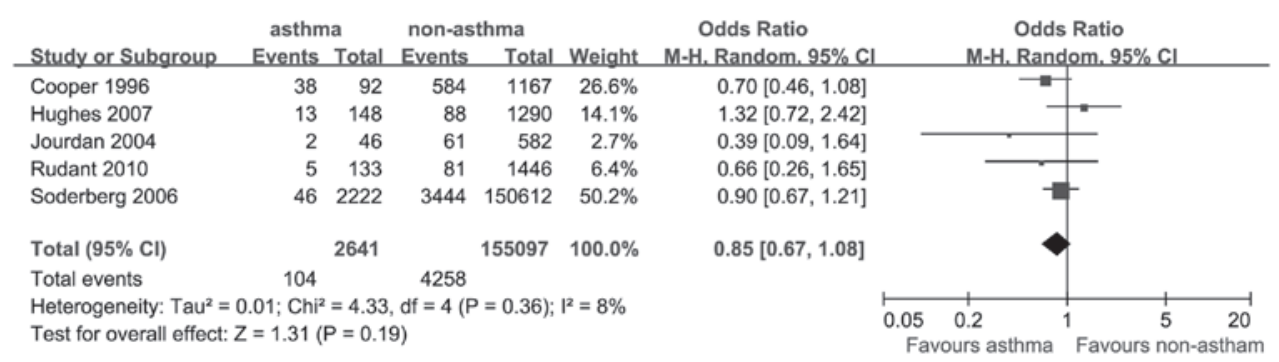

Figure 3. Estimates of the odds ratio of developing acute myeloid leukemia in patients with a history of asthma.

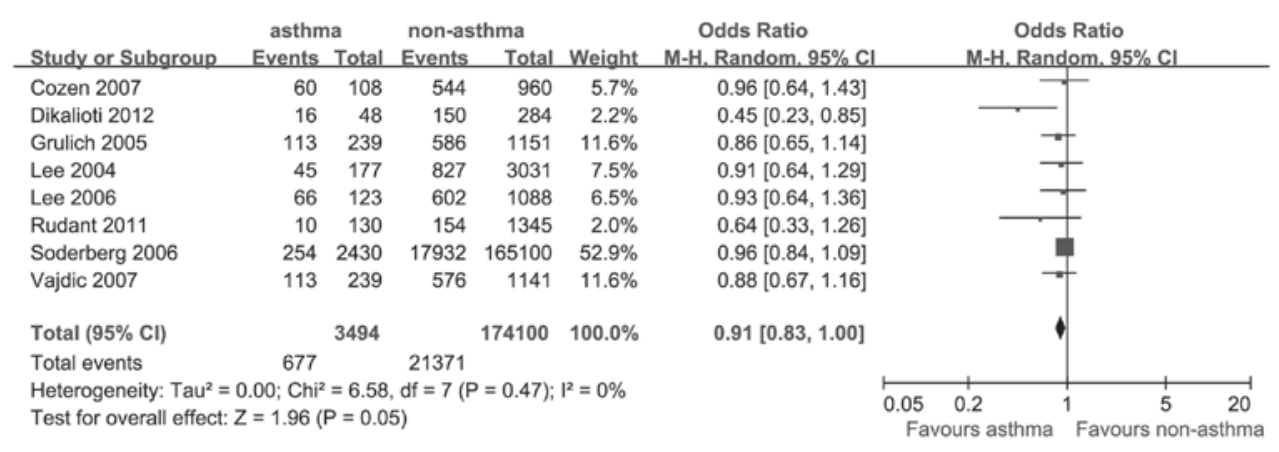

Figure 4. Estimates of the odds ratio of developing non-Hodgkin lymphoma in patients with a history of asthma.

malignancies (34). The occurrence of asthma may be a surrogate marker of an increased ability of the immune system to recognize and destroy malignant cells (23). By contrast, the antigenic stimulation hypothesis suggests that the chronic inflammatory state in asthmatic patients may result in an inflammatory cytokine cascade and lead to tissue damage and, ultimately, lymphoid malignancies (35). Asthma was associated with a reduced risk of NHL, which supports the immune surveillance hypothesis.

Our results revealed a non-significant odds ratio of 0.90 and 0.85 for the association of a history of asthma with ALL and AML, respectively,. The literature on asthma and acute leukemia is heterogeneous. In the present analysis, a $\sim 10 \%$ reduction in the risk for NHL in patients with a history of asthma was observed. An IgE-mediated anticancer response in asthma patients may be a possible explanation for the protective effect of asthma against the development of NHL (22). In addition, histamine, an inflammatory mediator in asthma, was reported to have the ability to defend normal cells by suppressing oxygen radical-induced damage and activating protective lymphocytes (36).
Asthma is considered as an overactive state of the immune response, characterized by a shift towards a Th2-dominant immunity. The persistence of a Th2-dominant immune system in asthmatic patients is inversely associated with the risk of NHL, of which the mechanism has not been fully elucidated. Other unmeasured factors associated with asthma may contribute to the reduced risk of NHL. Elucidating the mechanism of this negative association may provide valuable information on the effect of asthma on the immune response and the subsequent risk of developing NHL. The true underlying mechanism was not determined in the present analysis; however, our results support the immune surveillance hypothesis. We also hypothesize that genetic or exposure factors underlying the development of asthma may also be protective against NHL.

When analyzing the association between asthma and ALL, a high degree of heterogeneity was observed among the studies. A possible explanation for this severe heterogeneity may be the differences in the included studies regarding age range, country, number of cases and controls, diagnosis time 
and matching factors. Major differences in baseline characteristics largely account for the heterogeneity. By contrast, only mild heterogeneity and no heterogeneity were observed in the analysis of AML and NHL, respectively. Consequently, the results of the inverse association between asthma and NHL may be considered as credible.

The results of the meta-analysis should be interpreted with caution due to certain limitations. First, in the majority of the studies, the history of asthma was obtained from interview by telephone or questionnaire and recall bias may exist in the self-reported data $(37,38)$. Although the information bias appeared to be inevitable, the inverse association of asthma with acute leukemia and NHL (significant in NHL and non-significant in ALL and AML) was notable, provided that patients are more likely to pay attention to disease history, leading to information bias of a false-positive association (27). The positive association between asthma and the risk of developing acute leukemia or NHL was reported in previous studies $(17,18)$. Shared etiological factors may account for the results. However, as the reference date, corresponding with the date of diagnosis in cancer patients, is not well-defined in the controls, a history of asthma may be over-reported (31). This may lead to a higher incidence of asthma in the controls, from whom the data were obtained by interview. Previous studies reported only a moderate consistency between the medical reports and interview data (19). Second, there was no sufficient information on the morphological, immunological, genetic, molecular or pathological subtypes of acute leukemia and NHL in previous studies; therefore, further analysis of the association between asthma and the subtypes of these diseases was not feasible. Third, there was also a possible information bias in the data based on the medical records, as less severe cases of asthma may be not documented in medical records. This misclassification may exist equally among cases and controls, or more frequently in controls, resulting in a positive association trend of asthma with acute leukemia and NHL. Fourth, asthma-related medications may modulate the immune system, affecting the association of asthma with acute leukemia and NHL. The medical treatment of asthma may affect the risk of developing cancer due to cellular and humoral immunosuppression. In addition, the duration and severity of asthma, should also be taken into consideration. Fifth, confounding factors including age, gender and region of residence were controlled in the majority of the included studies, but other confounding factors, such as genetic characteristics, individual lifestyle, other immune-related diseases and infection exposure cannot be completely ruled out.

There were also several strengths in our study. First, the number of included cases and controls was sufficiently large to obtain a reliable association between asthma and ALL, AML and NHL. Second, the studies in our analysis included a diversity of countries and races, enabling the generalization of the outcomes. Third, with regard to NOS, all the studies included in our analysis were of high quality, ensuring reliable results. Fourth, in all the included studies, the cases and controls were matched by age and gender; region of residence and race were also matched in some studies, minimizing the potential information bias between cases and controls. Fifth, in the majority of the studies, a history of asthma was identified shortly before the diagnosis of acute leukemia or NHL.
Therefore, asthma during the early or indolent course of these diseases was excluded.

In conclusion, this study demonstrated that asthma is inversely associated with the incidence of NHL. A negative trend in the risk of ALL and AML in subjects with a history of asthma was also observed. Additional large prospective studies are required to elucidate the association between asthma and hematological malignancies.

\section{References}

1. Curado MP, Edwards B and Shin HR (eds): Cancer incidence in five continents. Vol 9. IARC Scientific Publications, Lyon, 2007.

2. Stiller CA and Parkin DM: Geographic and ethnic variations in the incidence of childhood cancer. Br Med Bull 52: 682-703, 1996.

3. Weidmann C, Black RJ, Masuyer E and Parkin DM: Incidence of non-Hodgkin's lymphoma in children between 1970 and 1990 in nine European countries. Eur J Cancer 35: 1235-1237, 1999.

4. Izarzugaza MI, Steliarova-Foucher E, Martos MC and Zivkovic S: Non-Hodgkin's lymphoma incidence and survival in European children and adolescents (1978-1997): report from the Automated Childhood Cancer Information System project. Eur J Cancer 42: 2050-2063, 2006.

5. Parker DM, Whelan SL, Ferlay J, Raymond L and Young J (eds): Cancer incidence in five continents. Vol 7. IARC Scientific Publications, Lyon, 2007.

6. Greaves M: Infection, immune responses and the aetiology of childhood leukaemia. Nat Rev Cancer 6: 193-203, 2006.

7. Urayama KY, Buffler PA, Gallagher ER, Ayoob JM and Ma X: A meta-analysis of the association between day-care attendance and childhood acute lymphoblastic leukaemia. Int J Epidemiol 39: 718-732, 2010.

8. Severson RK, Davis S, Thomas DB, Stevens RG, Heuser L and Sever LE: Acute myelocytic leukemia and prior allergies. J Clin Epidemiol 42: 995-1001, 1989.

9. Gallagher RP, Spinelli JJ, Elwood JM and Skippen DH: Allergies and agricultural exposure as risk factors for multiple myeloma. Br J Cancer 48: 853-857, 1983.

10. Bernard SM, Cartwright RA, Bird CC, Richards ID, Lauder I and Roberts BE: Aetiologic factors in lymphoid malignancies: a case-control epidemiological study. Leuk Res 8: 681-689, 1984.

11. McWhorter WP: Allergy and risk of cancer. A prospective study using NHANESI followup data. Cancer 62: 451-455, 1988.

12. Doody MM, Linet MS, Glass AG, et al: Leukemia, lymphoma and multiple myeloma following selected medical conditions. Cancer Causes Control 3: 449-456, 1992.

13. Jourdan-Da Silva N, Perel Y, Mechinaud F, et al: Infectious diseases in the first year of life, perinatal characteristics and childhood acute leukaemia. Br J Cancer 90: 139-145, 2004.

14. Rudant J, Orsi L, Menegaux F, et al: Childhood acute leukemia, early common infections and allergy: The ESCALE Study. Am J Epidemiol 172: 1015-1027, 2010.

15. Dikalioti SK, Chang ET, Dessypris N, et al: Allergy-associated symptoms in relation to childhood non-Hodgkin's as contrasted to Hodgkin's lymphomas: a case-control study in Greece and meta-analysis. Eur J Cancer 48: 1860-1866, 2012.

16. Vajdic CM, Falster MO, de Sanjose S, et al: Atopic disease and risk of non-Hodgkin lymphoma: an InterLymph pooled analysis. Cancer Res 69: 6482-6489, 2009.

17. Chang JS, Tsai YW, Tsai CR and Wiemels JL: Allergy and risk of childhood acute lymphoblastic leukemia: a population-based and record-based study. Am J Epidemiol 176: 970-978, 2012.

18. Spector L, Groves F, DeStefano F, et al: Medically recorded allergies and the risk of childhood acute lymphoblastic leukaemia. Eur J Cancer 40: 579-584, 2004.

19. Hughes AM, Lightfoot T, Simpson J, et al: Allergy and risk of childhood leukaemia: results from the UKCCS. Int J Cancer 121: 819-824, 2007.

20. Cooper GS, Kamel F, Sandler DP, Davey FR and Bloomfield CD: Risk of adult acute leukemia in relation to prior immune-related conditions. Cancer Epidemiol Biomarkers Prev 5: 867-872, 1996.

21. Soderberg KC, Jonsson F, Winquist O, Hagmar L and Feychting M: Autoimmune diseases, asthma and risk of haematological malignancies: a nationwide case-control study in Sweden. Eur J Cancer 42: 3028-3033, 2006. 
22. Cozen W, Cerhan JR, Martinez-Maza O, et al: The effect of atopy, childhood crowding and other immune-related factors on non-Hodgkin lymphoma risk. Cancer Causes Control 18 821-831, 2007.

23. Linabery AM, Jurek AM, Duval S and Ross JA: The association between atopy and childhood/adolescent leukemia: a meta-analysis. Am J Epidemiol 171: 749-764, 2010.

24. Smith CT, Williamson PR and Marson AG: Investigating heterogeneity in an individual patient data meta-analysis of time to event outcomes. Stat Med 24: 1307-1319, 2005.

25. DerSimonian R and Laird N: Meta-analysis in clinical trials. Control Clin Trials 7: 177-188, 1986.

26. Higgins JP and Thompson SG: Quantifying heterogeneity in a meta-analysis. Stat Med 21: 1539-1558, 2002.

27. Grulich AE, Vajdic CM, Kaldor JM, et al: Birth order, atopy and risk of non-Hodgkin lymphoma. J Natl Cancer Inst 97: 587-594, 2005.

28. Lee WJ, Cantor KP, Berzofsky JA, Zahm SH and Blair A: Non-Hodgkin's lymphoma among asthmatics exposed to pesticides. Int J Cancer 111: 298-302, 2004.

29. Lee WJ, Purdue MP, Stewart P, et al: Asthma history, occupational exposure to pesticides and the risk of non-Hodgkin's lymphoma. Int J Cancer 118: 3174-3176, 2006.

30. Rudant J, Orsi L, Monnereau A, et al: Childhood Hodgkin's lymphoma, non-Hodgkin's lymphoma and factors related to the immune system: the Escale Study (SFCE). Int J Cancer 129: 2236-2247, 2011
31. Schuz J, Morgan G, Bohler E, Kaatsch P and Michaelis J: Atopic disease and childhood acute lymphoblastic leukemia. Int J Cancer 105: 255-260, 2003.

32. Vajdic CM, Fritschi L, Grulich AE, et al: Atopy, exposure to pesticides and risk of non-Hodgkin lymphoma. Int J Cancer 120: 2271-2274, 2007.

33. Wen W, Shu XO, Linet MS, et al: Allergic disorders and the risk of childhood acute lymphoblastic leukemia (United States). Cancer Causes Control 11: 303-307, 2000.

34. Burnet M: Cancer: a biological approach. III. Viruses associated with neoplastic conditions. IV. Practical applications. Br Med J 1: 841-847, 1957.

35. Wang $\mathrm{H}$ and Diepgen TL: Is atopy a protective or a risk factor for cancer? A review of epidemiological studies. Allergy 60: 1098-1111, 2005.

36. Hellstrand K: Histamine in cancer immunotherapy: a preclinical background. Semin Oncol 29: 35-40, 2002.

37. Gail MH and Benichou J: Encyclopedia of epidemiologic methods. John Wiley \& Sons Ltd., New York, 2000.

38. Infante-Rivard C and Jacques L: Empirical study of parental recall bias. Am J Epidemiol 152: 480-486, 2000. 